¿Que-es-Para-ti-la-eDucación?
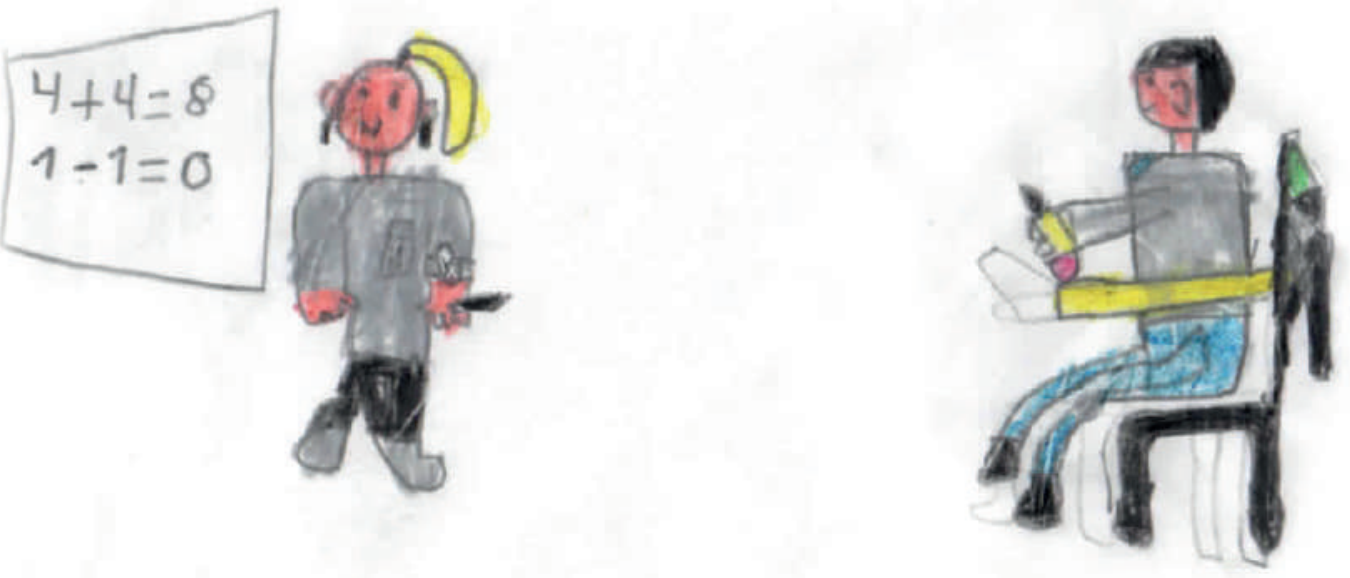

Ser educado.

Estudios deDerecho

UNIVERSIDAD

Fundada en 1912

DE ANTIOQUIA 


\title{
Flexibilizar la gestión administrativa del Estado colombiano en tiempos de globalización
}

\author{
José Ignacio González Buitrago**
}

El Estado es cada vez más inoperante en loglobaly
cada vez menos representativo en lonacional.

Manuel Castells

\begin{abstract}
Resumen
El estudio identifica los mecanismos jurídico-administrativos que el Estado colombiano ha implementado o debe implementar para hacer su estructura administrativa más flexible y, en consecuencia, más efectiva, eficiente y garantista. Describe cómo operan cada uno de ellos y, a su vez, observa el cumplimiento de los compromisos que debe acatar el Estado en el marco de los vigentes procesos de internacionalización y globalización. En tres grandes acápites se hace referenciaa la delimitación de losconceptos de globalización, sociedad de la informacióny sociedad del conocimiento, para luego asumir la modernización del Estado desde dos aspectos: el nuevo enfoque de la gerencia pública y el concepto de gobierno electrónico (e-gobierno) o gobierno en línea, para el caso colombiano. Finalmente se describen los mecanismos jurídico-administrativos que optimizarían la flexibilización de la gestión y estructura del Estado. En este punto, se dedica una sección a determinar los dispositivos juridico-administrativos implementados por el gobierno colombiano dentro de la estrategia de Gobierno enlínea.

Palabras Clave: flexibilización de la gestión del Estado; gobierno electrónico; gobierno en línea; globalización, modernización del Estado; las TIC en la administración pública.
\end{abstract}

\section{Flexibilizing the administrative management of Colombian state in time of globalization}

\begin{abstract}
The study identifies the legal-administrative mechanisms that the Colombian State has implemented or must implement to make its administrative structure more flexible and, consequently, more effective, efficient and guarantor.It describes how each ofthem operatesand, atthesame time, observesthe fulfillment of thecommitments that the State must comply with within the framework of the current processes of internationalization and globalization. In three major sections reference is made to the delimitations of the concepts of globalization, the information society and the knowledge society, to then assume the modernization of the State from two aspects: the new approach of public management and the concept of electronic government (e-government) or online government, for the Colombian case. Finally, the legal-administrative mechanisms that would optimize the flexibility of the management and structure of the State, are described. At this point, a section is dedicated to determining the legal-administrative devices implemented by the Colombian government within the online Government strategy. Keywords: flexibilizing State administrative management; Electronic government; online government; globalization; modernization of the State; ICT in public administration.
\end{abstract}

\section{Flexibilizar a gestão administrativa do Estado colombiano em tempos de globalização}

\section{Resumo}

Este estudo identifica os mecanismos jurídico-administrativos que o Estado colombiano implementou ou deve implementar para tornar a sua estrutura administrativa mais flexível e, consequentemente, mais efetiva, eficiente e garantista. Descreve a maneira como funciona cada um deles e, ao mesmo tempo, observa o cumprimento dos compromissos que o Estadodeve obedecer no quadrodosprocessosvigentes deinternacionalização eglobalização. Em três grandes parágrafosfaz-se referênciaà delimitação dosconceitosdeglobalização, sociedade da informaçãoe sociedade doconhecimento, para depoisassumir amodernizaçãodo Estadodesde doisaspectos: a novaabordagem da gerência pública e o conceito de governo eletrônico (e-governo) ou governo em linha, para o caso colombiano. Finalmente, descrevem-se os mecanismos jurídico-administrativos que otimizariam a flexibilização da gestão $e$ estrutura do Estado. Neste ponto, dedica-se uma seção para determinar os dispositivos jurídico-administrativos implementados pelo governo colombiano na estratégia de Governo em linha.

Palavras-chave: flexibilização da gestão do Estado; governo eletrônico; governo em linha; globalização; modernização do Estado; as TIC na administração pública.

* Artículo de reflexión. El presente artículo presenta uno de los productos de los resultados de la investigación terminada sobre sobre Gobierno Electrónico, desde una perspectiva analítica, interpretativa y crítica sobre este tema específico, desarrolla da por José Ignacio González Buitrago, en la convocatoria 2015 - 2016, de la Universidad La Gran Colombia, en donde presta sus servicios como docente - investigador de la Facultad de Postgrados, Área de Derecho. La línea de investigación que coincide con el nombre del grupo, denominada: "Derecho constitucional, reforma de la administración de justicia y Bloques deConstitucionalidad".

** Magister en Dirección Universitaria de la Universidad de Los Andes, Especialista del Lenguaje Audiovisual de la Universidad El Bosque, abogado de la Universidad Pontificia Bolivariana y director del Centro de Conciliación de la Fundación Pluma en la ciudad de Bogotá, Colombia..Correo electrónico: joseignaciog018@yahoo.es. ORCID:00000002-1872-124X

Cómo citar este artículo: González Buitrago, J. E. (2019). Flexibilizar la gestión administrativa del Estado colombiano 


\section{Flexibilizar la gestión administrativa del Estado colombiano en tiempos de globalización}

\section{Introducción}

El uso de las Tecnologías de la Información y la Comunicación (en adelante las TIC) en la gestión administrativa de los Estados ha exigido de estos que se produzcan marcos normativos que permitan su incorporación a las dinámicas propias de la modernización y de la organización de los mismos.

La inserción de las TIC en la vida gubernamental facilita la relación entre administración y administrados y con ello el dar cumplimiento a los principios constitucionales de celeridad, eficacia y economía, propios de la actividad administrativa del Estado.

La presente investigación se enmarca dentro del fenómeno de la globalización, en el cual los gobiernos se obligan a implementar estrategias que posibiliten una estructura administrativa flexible, sin desconocer los principios constitucionales y legales que regulan su actuación. Esta situación implica una búsqueda de la optimización, tanto de recursos económicos, como de tiempo que el Estado emplea para das respuesta oportuna a las inquietudes de los ciudadanos sobre los servicios públicos ofrecidos directamente por la administración. Ante este imperativo, las TIC se constituyen en mecanismos idóneos para mejorar la prestación de los servicios gubernamentales.

Es común escuchar a los ciudadanos expresarse sobre el Estado como una organización rígida, lenta, cargada de trámites y propensa a la corrupción. Estos fenómenos ha sido objeto de estudio de las Naciones Unidas, que en consecuencia ha solicitado a los Estados miembros que revisen su normatividad e implementen cambios que permitan incorporar las nuevas TIC en el manejo de los asuntos públicos y se ajusten a las exigencias propias de la globalización. Es así como cada país, dentro de las discusiones en las conferencias sobre el gobierno electrónico y en el marco de la celebración y aprobación de los tratados de libre comercio, han adquirido compromisos ineludibles sobre la modernización de los entes guber- 
namentales. Posiblemente, si la organización y gestión del Estado se flexibilizara, implementando mecanismos tales como el gobierno electrónico, que implica la utilización de las nuevas tecnologías para acceder a los servicios del Estado y la incorporación social de la información propia de la sociedad del conocimiento, la relación Estado y sociedad sería más cercana y obtendría mejores resultados dentro de la perspectiva de una democracia real.

Son muchas las perspectivas desde las cuales se puede analizar el tema propuesto. El presente trabajo de investigación busca una respuesta a la pregunta ¿cuál o cuáles son los mecanismos administrativos que el Estado colombiano debe implementar para lograr la flexibilización de su estructura administrativa, en tiempos de globalización? Con esta pregunta se busca identificar los mecanismos jurídicos administrativos que el Estado colombiano debe implementar para hacer su estructura administrativa más flexible y, una vez superada esta etapa, pasar a describir cómo operan cada uno de ellos y finalmente, observar si con ello se cumple con los compromisos internacionales, relacionados con la inserción del país en los procesos de globalización.

La implementación de las TIC en la organización de los Estados se constituye en un instrumento trascendental para lograr un nuevo diseño de los procedimientos administrativos, o como lo denomina las Naciones Unidas, el diseño del gobierno electrónico (e-gobierno).

El surgimiento del gobierno electrónico, podría propiciar, entre otras, una mayor transparencia en las actuaciones de los servidores públicos; mayor eficacia y eficiencia en los trámites; mejores respuestas a los ciudadanos; reducción en el tiempo de respuesta y, por supuesto, permitiría generar confianza de la colectividad en el sistema, al mejorar la calidad en la prestación de los servicios.

En el caso particular de Colombia, los objetivos que se pretenden con la implementación del gobierno electrónico o gobierno en línea, como se denomina en el país, solo podrán cumplirse si se desarrollan con plena concordancia con los principios constitucionales sobre: "Los Fines del Estado" y los "Principios de la Función Pública”, artículos $2^{\circ}$ y 209, ampliados en la Ley 489 de 1998, Por medio de la cual se reglamenta la función administrativa", artículo $3^{\circ}$, en los siguientes términos: "La función administrativa se desarrollará conforme a los principios constitucionales, en particular los atinentes a la buena fe, igualdad, moralidad, celeridad, economía, imparcialidad, eficacia, eficiencia, participación, publicidad, responsabilidad y transparencia" (Colombia, Congreso Nacional, 1998).

En razón de lo anterior, los resultados que se presentan en el presente trabajo permitirán entender y conceptualizar cómo las TIC sirven de mecanismo jurídico para hacer más efectivos los servicios que debe prestar la administración pública en cumplimiento de los principios constitucionales y legales. 


\section{Antecedentes}

Durante la década de los noventa del siglo XX, en América Latina, se impuso la implementación de reformas en la estructura y gestión administrativa de los Estados, originadas en las exigencias propias del llamado "Consenso de Washington", y que sirven de puerta hacia la aparición del concepto neoliberal sobre el Estado que propende porque la economía de mercado sea la que oriente la relación Estadomercado en el continente americano. Frente a esto, la mayoría de los gobiernos de la región emprendieron programas de ajuste estructural de la administración con el deseo de mejorar la calidad de la democracia y evitar el enfrentamiento entre el Estado y ciudadanía, que tiene su origen en la existencia de altos niveles de pobreza y la desigualdad existente en la mayoría de los Estados de América Latina.

Para la segunda mitad de la década de los noventa, durante el siglo XX, aparece un segundo elemento impuesto por organismos internacionales consistente en la obligación de adoptar políticas públicas que permitan el uso de los adelantos de las TIC para propiciar que la acción del Estado sea más cercana al ciudadano y que sus acciones faciliten la participación ciudadana en los asuntos públicos. Esto crea espacios en los que todos se comprometen y aportan para mejorar los servicios que el Estado ofrece a la comunidad en general. En consecuencia, se da inicio a unos primeros intentos al reducir los procesos administrativos, con normas antitrámites; disminución en el tiempo de respuesta a las solicitudes de los ciudadanos, con la creación de las Ventanillas Únicas y optimizar la seguridad en la circulación de los documentos públicos al ofrecer los servicios de consulta, por medio de las páginas web de las entidades públicas. Intentos que vistos hoy desde la perspectiva del avance de la tecnología, parecieran insignificantes. Sin embargo, estos esfuerzos han derivado en grandes transformaciones en la cultura institucional de los gobiernos latinoamericanos, al hacer más eficiente y transparente la gestión gubernamental.

En el año 2004, un informe de las Naciones Unidas, elaborado dentro del marco del Programa de Naciones Unidas para el Desarrollo (PNUD), denominado La Democracia en América Latina: Hacia una democracia de ciudadanas y ciudadanos, orientado por el profesor Guillermo O'Donnel, se constituye en un acontecimiento trascendental para el futuro de lo que se llegaría a denominar e-gobierno.

Es indudable que las nuevas tecnologías han producido un cambio radical en la sociedad, haciendo de ella una verdadera sociedad de la información y del conocimiento. A su vez, este avance ha propiciado la existencia de soportes de información y sistemas de representación que han creado vínculos económicos y políticos dentro de las sociedades que los utilizan.

María Belén Albornoz (2006) resume la implementación del gobierno electrónico como aquel fenómeno que surge a fines de los años noventa, de una parte, 
como el resultado de procesos de incorporación de la tecnología por parte de la sociedad, y por otro lado, debido a factores propios de la última década del siglo $\mathrm{XX}$, tales como el nacimiento de la sociedad de la información, la globalización económica y cultural, el debilitamiento institucional y funcional de los EstadosNación, los procesos de descentralización estatal y las nuevas formas de experimentar con la democracia directa.

Más adelante indica: "El concepto de Gobierno Electrónico es rápidamente apropiado en América Latina por los gobiernos locales, en especial por los municipios, considerando que Internet puede ser una herramienta útil de gobernabilidad" (Albornoz, 2006, p.19).

\section{Delimitación de los conceptos: globalización, sociedad de la información y sociedad del conocimiento}

Con el objeto de asumir el planteamiento del problema que se ha propuesto en la investigación, a continuación se hace una aproximación a los conceptos de globalización, sociedad de la información y sociedad del conocimiento, para luego observar sus relaciones e implicaciones en la organización del Estado.

\section{La globalización}

En términos generales y siguiendo lo dicho por Manuel Castells (1998), simplemente, iniciemos preguntándonos ¿qué es la globalización? Y este autor nos dice:

Es el proceso según el cual las actividades decisivas en un ámbito de acción determinado (la economía, los medios de comunicación, la tecnología, la gestión del medio ambiente, el crimen organizado) funcionan como unidad en tiempo real en el conjunto del planeta. Se trata de un proceso históricamente nuevo (distinto de la internacionalización y de la existencia de una economía mundial) porque solo en la última década se ha constituido un sistema tecnológico (telecomunicaciones, sistemas de información interactivos, transporte de alta velocidad en un ámbito mundial para personasy mercancías) que hace posible dicha globalización. La informacionalización de la sociedad, a partir de la revolución tecnológica que se constituye como nuevo paradigma operativo en la década de los setenta, es la base de la globalización de la economía [...]. (p.2)

No obstante, frente a la pretensión de definir el fenómeno de la globalización, es innegable que el concepto es vago y difícil de concretar. Generalmente se escucha hablar de la globalización refiriéndose a fenómenos diversos, distantes unos de otros. 
No todo, la vida de las naciones es global, por ejemplo, las instituciones públicas de naturaleza nacional son las que establecen las políticas públicas sobre el empleo, la economía, la vida en sociedad y las formas de participación de la ciudadanía. Siendo esto cierto, se debe aceptar que los procesos estructurantes de las economías locales, de la tecnología y del uso de las comunicaciones son cada vez más globales.

Un aspecto importante para resaltar es el que tiene que ver con la globalización como un sistema que funciona haciendo uso de la red, que a su vez, hace uso de la tecnología de la información e influye en todo aquello que sea de utilidad para los grandes conglomerados financieros, de capitales, de publicidad y para el movimiento de grandes volúmenes de mercancía entre los países; haciendo de la competencia su mayor expresión. En este escenario:

La globalización es a la vez dinamismo productivo, inclusión de los creadores de valor, y marginación social, exclusión de quienes carecen de interés como productores o consumidores desde la perspectiva de productividad, competitividad y ganancia, que se constituyen en el criterio fundamental para mercados desregulados y economías privatizadas. (Castells, 1998, p.2-3)

Los Estados, dentro del actual proceso de globalización, tienen, igualmente, la obligación de estar inmersos en los sistemas financieros internacionales y acoger sus disposiciones; lo que implica la creación de políticas públicas que propicien la llegada de multinacionales o de capital financiero a los países. Estar dentro de las reglas de juego de los mercados internacionales genera algunos problemas, dentro de los cuales se pueden señalar: i) en primer lugar, el Estado se obliga a asegurar márgenes de utilidad para los inversionistas que son atraídos por las nuevas condiciones del mercado, en detrimento de los inversionistas internos. También puede comprometer mayores costos para las entidades estatales; ii) en segundo lugar, se pueden suscitar movimientos especulativos en las bolsas de mercado, que pueden generar inestabilidad económica en el país, no controlable por los bancos centrales, al no obedecer propiamente a criterios económicos sino a manipulaciones informativas.

El poder real de las redes globales empresariales está en los mercados financieros que controlan las grandes corporaciones, orientan las organizaciones multilaterales y constriñen a los gobiernos nacionales para someterlos a su voluntad insaciable para lograr el máximo beneficio utilitario. (Barone, 2001, p.15)

\section{Sociedad de la información}

El acompañamiento de las TIC, en la vida cotidiana de las personas, ha motivado un cambio revolucionario y ha dado lugar a la denominada "sociedad de la in- 
formación", en la que la información juega un papel estratégico como elemento de poder cada vez más preponderante (Castells, M, 1996). Esta transformación ha repercutido en una serie de aspectos relacionados con el manejo de la información, que sin duda, tienen una incidencia jurídica. Dentro de esta incidencia se puede destacar: el incremento exponencial de la producción documental y la evolución imparable de las tecnologías empleadas para la creación y gestión de estos documentos.

Sobre el origen de la denominación sociedad de la información, es internacionalmente aceptado que surgió como consecuencia de dos documentos: el primero denominado Crecimiento, competitividad y empleo. Retos y pistas para entrar en el siglo XXI, realizado por la Comisión de Comunidades Europeas en el año de 1993 y el segundo, Europa and The Global Information Sicuety, que es un conjunto de recomendaciones del Consejo Europeo realizadas en 1994. En estos documentos se trata de definir el concepto y otorgarle algunas características. En este sentido es importante destacar la investigación de los profesores Cortés Sánchez, J. y Cardona Madariaga, D. (2015) realizada en la Universidad El Rosario, destacan este hecho y muestran los verdaderos alcances de ambos documentos y la importancia para el desarrollo del concepto sobre sociedad de la información.

Con relación al documento Europa and The Global Information Sicuety, tiene un aspecto importante para resaltar, se trata de un llamado a la cooperación entre los países europeos y una invitación a trabajar unidos el sector público y privado, con el objeto de integrar a Europa a la sociedad del conocimiento aprovechando todos los beneficios que trae la combinación sociedad y tecnología, en donde la participación ciudadana, la innovación, la competitividad del sector privado, la transparencia de los mercados, entre otros, propicie el crecimiento económico (Cortés, et al., 2015).

De acuerdo con la Comisión Económica para América Latina y del Caribe CEPAL (1995), la incursión de nuevas tecnologías de información y comunicación en el mercado ha provocado cambios de trascendental importancia en la vida cultural, social y económica de los países y consecuentemente en las formas de administración de las organizaciones públicas, privadas, de producción, de trabajo, de diversión; cambios que están modificando notablemente estas organizaciones, razón por la cual las estrategias también deben cambiar.

La existencia de las autopistas de la información permite identificar grandes cambios en las actividades diarias de todas las personas, como por ejemplo, la oferta de información puesta a disposición de quien la necesite, sin barreras ni censuras. Esto ha hecho que se identifiquen nuevas formas de trabajos productivos, de relacionarse con los demás, de divertirse y de adquirir bienes y servicios; generando de paso, una cultura de consumo informático. 
Los desarrollos tecnológicos de las telecomunicaciones y la computación enriquecen, a la vez que hacen más complejo el panorama del campo de la información. El mejoramiento de las capacidades de los equipos computacionales, el aumento de las capacidades de almacenamiento de información en revolucionarios soportes y la expansión geográfica de redes electrónicas, configuran una oferta tecnológica para las actividades de información que incluso superan las posibilidades actuales de utilización exhaustiva de las redes de información de la región. (CEPAL, 1995, p.6)

Los Estados requieren grandes inversiones en equipos para incorporarse a la modernización tecnológica, pero no solo esto asegura un resultado positivo, adicionalmente, se deben desarrollar políticas públicas que hagan posible la capacitación del talento humano, tanto de las organizaciones públicas, como de las privadas; la superación del analfabetismo funcional informático de los ciudadanos en general y el rediseño de procesos y estructuras del Estado, que permitan el funcionamiento de la red, como mediadora de la relación entre el ciudadano y el Estado. Como dice Isaac Enríquez Pérez (2002):

Una política pública y social que deje de lado estas nuevas necesidades y nuevos actores queda impedida para habilitar a los individuos de las naciones latinoamericanas para enfrentarse a las ventajas y desafíos derivados del proceso de globalización del capital y la de la 'sociedad del conocimiento' que en una de sus vertientes la impulsan. (p.40)

Las nuevas tecnologías han dado un paso más adelante, al articular sobre internet y la telefonía móvil, lo que se podría denominar "web social", que permite a cualquier ciudadano organizar comunicaciones en grupo o redes de expresión, colaboración o de contenidos que han dado muestra de efectividad; esto es solo posible por las aplicaciones que las compañías de comunicaciones ponen a su disposición.

\section{Sociedad del conocimiento.}

En la segunda mitad del siglo XX la sociedad vio nacer la radiodifusión de microondas, la televisión por cable, las comunicaciones satelitales, los equipos de cómputo, el teléfono móvil, la internet y las aplicaciones tecnológicas que supusieron, en su momento, cambios de gran impacto en las comunicaciones. Todo ello sirvió para afirmar los cambios paradigmáticos que se presentaron en los hábitos de vida de millones de personas, en las postrimerías de la pasada centuria. Como resultado de esos cambios, en la última década, se anunció el nacimiento de la llamada "sociedad de la información" definida por Peter Drucker (1974) como: "aquella agrupación social para quienes la información y el conocimiento se convierten en 
la principal fuente de poder". Las ventajas que representan las nuevas tecnologías de la información, residirían, según sus propulsores -no pocos- en que éstas han alcanzado, gracias a las comunicaciones, todos los públicos, los medios sociales y los ámbitos culturales, diferenciándose significativamente de otros medios, en el hecho de que los usuarios de las mismas se relacionan en tiempo real.

De lo dicho en el párrafo anterior se desprende que, cuando se hace referencia a la sociedad del conocimiento, de inmediato se trae a colación los grandes flujos de información que proporcionan las redes de comunidades locales, nacionales o globales. "[D]e hecho, en todas las áreas principales de la política, la interconexión de las comunidades políticas nacionales en los flujos y procesos regionales y globales las hace tomar parte en una intensiva coordinación y regulación transfronterizas" (Velázquez, C. \& Pérez, G., 2010, p.126).

Los medios de difusión y asimilación de la información, a través de estructuras interactivas, son una realidad dado el aporte brindado por las tecnologías de la información y la comunicación. Estas facilitan la construcción de redes interpersonales de sujetos unidos por intereses comunes o la alianza de personas vinculadas por aficiones y deseos, que los motivan y convocan a movilizaciones o acciones colectivas que trascienden la individualidad de cada uno de los participantes.

El Estado no ha sido ajeno a las grandes transformaciones producidas por la incursión de las prácticas globalizadas y la utilización de grandes flujos de información, de allí que sea creciente la importancia de las consecuencias de estos fenómenos que afectan directamente las perspectivas de su uso, para hacer posible la democracia y su ejercicio, el ejercicio de la ciudadanía democrática, la igualdad.

\section{Modernización del Estado}

Antes de plantear la necesidad de transformación que tiene el Estado moderno, dentro del contexto de la globalización, es pertinente, en primer lugar, conocer y ver los alcances del ámbito de lo público para luego abordar la temática relacionada con los procesos de modernización del Estado; de manera general y eminentemente conceptual. El ámbito de lo público es, en abstracto, un espacio en el que convergen las nociones de Estado, Nación y Sociedad Civil, para promover el bien general, el bienestar para todos y el desarrollo social y humano.

En este contexto, el Estado deberá asumir un nuevo rol que lo conecte, lo articule, de forma efectiva, con la sociedad. Pero esto no se logra simplemente implementando en lo público los modelos de gestión de las organizaciones empresariales privadas, pues estas solo tienen como horizonte el lucro. Por el contrario, 
el Estado no está para generar rendimientos económicos, por el contrario, está para dar rendimientos sociales y ello lo puede lograr con modelos de gestión más eficientes y eficaces que contribuyan a dar soluciones a los problemas que le son apremiantes al grueso de la sociedad. Posiblemente los modelos privados no sean el mejor camino, el Estado tiene su propia forma de ejercer la administración pública. Al respecto afirma Álvaro Navarro (1998):

En esa dirección, hay que partir de una premisa fundamental: El Estado no escoge sus clientes o usuarios, sino que por definición está al servicio del bien común. El Estado es un producto social, por lo tanto lo social solo tiene sentido en relación con el Estado en la medida que este corresponda y responda a los intereses de toda la sociedad que muchas veces son contradictorios y antagónicos entre sí. (p.6)

La globalización ha generado la percepción de que el poder efectivo ya no se encuentra únicamente en los Estados nacionales, por el contrario, que fuerzas y actores que operan en el ámbito nacional, regional e internacional comparten ese poder efectivo y lo intercambian. Según Velázquez C. \& Pérez, G. (2010):

La actuación de los Estados en sistemas regionales y globales cada vez más complejos afecta tanto su autonomía -al alterar los costos y los beneficios de las políticas y al influir en los programas institucionales- como su soberanía -al cambiar el equilibrio entre las estructuras jurídicas y las prácticas administrativas nacionales, regionales e internacionales. (p.126)

Estos mismos autores aclaran, de forma más puntual, la afirmación que se ha acuñado ya hace varios años en la teoría política, y que surge como consecuencia de las críticas al fenómeno de la globalización y la proliferación de la firma de tratados de libre comercio, así como las consecuencias que estos traen para los países. Lo expresan así:

La interconexión regional y global desafía tanto la soberanía como la legitimidad de los Estados. Son un peligro a la soberanía porque la autoridad política de éstos se ve desplazada y comprometida por los sistemas regionales y globales de poder, ya sean políticos, económicos o culturales. Y son un reto a la legitimidad del Estado porque, con la mayor interdependencia regional y global, éstos no pueden suministrar bienes y servicios fundamentales a sus ciudadanos sin la cooperación internacional, y aun esta última puede resultar bastante inadecuada frente a problemas globales -desde el calentamiento global hasta los volátiles movimientos de los mercados financierosque pueden escapar a la regulación política. (Velázquez, C. \& Pérez, G. 2010, págs. 112, 113) 


\section{Nuevo enfoque de la gerencia pública}

Con el proceso de modernización del Estado corre paralela la visión de una nueva gerencia pública, por ser complementarios como procesos inacabados. En particular, la nueva gerencia pública surge en la medida que el ente público esté dispuesto al cambio para responder a las necesidades que plantea la sociedad. Se requiere organizaciones flexibles y con capacidad de adaptación y, por supuesto, con gerentes públicos innovadores, que generen espacios de participación democrática en donde se permita la creación de redes que faciliten la toma de decisiones y la implementación de políticas públicas. Lo anterior será posible materializarlo solo si se crea, por parte del Estado y los administradores públicos, una nueva cultura de responsabilidad en la prestación del servicio público, acompañada de nuevos valores en torno a lo que es el Estado y su función frente a los particulares.

Modernizar el Estado en éste y todos los casos es mucho más que el acceso a las tecnologías de información y a las técnicas de management; reducción del déficit fiscal y reducción del gasto público. Es sobretodo centrar la atención en la calidad de las personas que conforman las organizaciones para que manifiesten su creatividad e innovación en la producción de bienes y servicios. (Navarro, 1998, p.7)

Desde el ámbito de lo público, es posible hoy visualizar los grandes desafíos que afrontaran los actuales y futuros gobernantes, en el proceso de modernizar el Estado. Estos tienen como reto principal, propiciar políticas públicas que fomenten una relación fluida entre el gobierno y el sector privado, de tal forma, que haciendo uso de las Tic dentro de la estrategia pública del gobierno electrónico o gobierno en línea se promueva el crecimiento económico, y de paso, se consoliden procesos democráticos.

De acuerdo con la CEPAL (1998) este aspecto muestra que:

No obstante, las transformaciones que se producen al interior del Estado y por consiguiente en la sociedad, empiezan a producir impactos positivos en los niveles de productividad, eficiencia en los servicios, actualización tecnológica que agiliza los procesos burocráticos y una serie de otros sucesos que en la reformulación del rol del Estado, se señala como un profundo redimensionamiento del aparato estatal y, al mismo tiempo, un mejoramiento sustancial de la efectividad del Estado como ente capaz de regular la transformación productiva y de liderar en el logro de la equidad social. (pág.10)

En el fondo, no solo la estructura del Estado es la que ha de democratizarse, sino que es la sociedad en general la que, con la ayuda de la información y del 
conocimiento, facilitada por las TIC, debe conseguir una mayor y real posibilidad de participación en los destinos del país.

Para hacer converger los intereses de la sociedad en su conjunto y dar sentido al término ciudadanía se torna necesario el fortalecimiento del sujeto popular y las organizaciones sociales. El desmantelamiento del Estado, entendido como la reducción de sus obligaciones frente al ciudadano, lleva a estos grupos a actuar al margen de él en la búsqueda de respuestas a sus demandas legítimas. Pero para que no queden convertidos en hechos aislados, es imprescindible que entre estos movimientos haya cierta aglutinación, coordinación o conjunción que desemboque en una redefinición del Estado que garantice el desarrollo social. Nueva construcción en la que el poder debe desconcentrarse y otorgarse mayor participación a la sociedad, lo que revestiría de sentido a los órganos gubernamentales representativos, hoy desvirtuados. Como así también daría cabida a un sujeto reformista y autónomo que haría valer su fragmentación como una forma de vehicular los distintos intereses de los grupos sociales. (Barone, 2001, p.12)

\section{Gobierno electrónico - E-gobierno - Gobierno en línea}

El origen del "gobierno electrónico" se puede rastrear desde finales de la década de los setenta, cuando surgió la discusión sobre las reformas que demandaba la administración pública, tema que desencadenó una serie de reflexiones sobre cuál era el mejor modelo para hacer eficiente la organización del Estado y situarlo más cercano a la comunidad. Sobre este escenario se presentaron dos corrientes: la primera tiene su origen en el neoistitucionalismo y la segunda corresponde a la corriente sobre la nueva gestión pública. Esta última incluyó, dentro de sus reflexiones, la incorporación paulatina de herramientas informáticas al servicio de la organización estatal. La corriente neoinstitucional, con relación a las nuevas tecnologías de la información y la comunicación, se debatía sobre si el impacto de estas era o no positivo debido a la brecha entre los avances tecnológicos propiamente dichos y la falta de capacitación de los servidores públicos sobre el manejo de dichas tecnologías.

La globalización y sus efectos en todos los países del continente americano produjeron varios cambios, en especial los que se refieren al sector de las telecomunicaciones, que muestran una verdadera revolución tecnológica representada en la entrada en operación de internet, con múltiples aplicaciones en la economía, el comercio, la cultura y la educación. Así mismo, se desarrolló el sistema de telefonía móvil. Ese mundo digital será la base para el auge y consolidación de la sociedad tecnológica, desde donde se proyecta el futuro de la administración pública y el 
acercamiento de los ciudadanos al Estado, ampliando el espectro de la participación ciudadana en la búsqueda de garantías sociales, comunitarias e individuales.

Retomando conceptos desarrollados por el profesor Castells (1996), la información juega un papel estratégico cada vez más importante como elemento de poder. En razón de lo anterior, no es extraño que la transformación generada por la información y las nuevas tecnologías asociadas a ella, haya repercutido en una serie de aspectos, entre los más notables se encuentra lo relacionado con el manejo de la información en la vida pública y privada, el manejo de bases de datos, los adelantos de la telefonía y los servicios incorporados a ella tales como el correo electrónico (tener una o varias cuentas personales de correo), la consulta en tiempo real de información requerida o la prestación de servicios estatales de información. Todos estos desarrollos con incidencia en el derecho, la administración pública y la posibilidad de hacer uso de ellos para realizar una oportuna y segura participación en la vigilancia y control de la organización del Estado, como instancia de regulación de la vida en sociedad.

El Estado no se ha quedado atrás con respecto al uso de la información y las telecomunicaciones y los ha puesto a su servicio haciendo posible, en primera instancia, el manejo de la información para acercar las comunidades a la gestión administrativa adecuando los procesos y la estructura administrativa al logro de los propósito de eficiencia y efectividad, que son cometidos estatales ordenados por la Constitución en su artículo segundo.

La incorporación de las TIC en la administración estatal ha generado una nueva visión de lo público y ha propiciado la modernización de la esfera pública. A este fenómeno se le ha denominado "administración electrónica" o según los organismos internacionales "gobierno electrónico" o "e-gobierno"1. El origen de la expresión Electronic Government (gobierno electrónico) se encuentra en dos informes originados en los Estados Unidos de América a finales de la década de los noventa y surgen dentro de un contexto de diagnóstico sobre el uso de las TIC en los procesos de modernización del Estado. Estos informes son: The Nacional Performence Review y Nacional Information Infraestructure, (Informe Nacional del Funcionamiento de la Infraestructura Nacional de Información) y un segundo denominado Nacional Partnership for Reinventing Government (Asociación Nacional para Reinventar el Gobierno).

En el caso particular de Colombia, durante los años noventa, investigadores nacionales e internacionales al analizar el desempeño del país dentro del contexto internacional coincidían en que presentaba deficiencias notables en el desarrollo

1 Otras denominaciones del "gobierno electrónico" son: “E-governance”, “E-government”, gobierno digital, gobierno en línea, teleadministración, one-stop goverment, electronic government, online government. Cada país adopta el nombre que más represente esta forma de modernización de la administración pública a través del uso de internet. 
de las telecomunicaciones y, consecuentemente, la aplicación de los servicios ofertados por la administración pública eran igualmente precarios.

Desde la perspectiva internacional es importante destacar la labor de los organismos multilaterales como Naciones Unidas (ONU), la Comisión Económica para América Latina y el Caribe (CEPAL), el Instituto Latinoamericano y del Caribe de Planeación Económica y Social (ILPES) y la Organización para la Cooperación y Desarrollo Económico (OCDE), entre otros. Ellos han propiciado conferencias en donde se describen experiencias exitosas, que por sus efectos son compartidas a los países miembros, los cuales adquieren el compromiso de ponerlas en marcha. Seguidamente, se identifican algunas de estas reuniones, en particular, las que han impulsado el uso de las TIC en el proceso de modernización del Estado.

El Consejo Económico y Social de las Naciones Unidas convocó en el año 2000 a los Estados miembros para que participaran en una conferencia de alto nivel sobre la promoción de una administración moderna. Los Representantes de América Latina y el Caribe se reunieron para preparar su participación y suscribieron la Declaración de Florianópolis, en donde se establece como una de las líneas de acción la siguiente:

Promover una administración más eficiente, eficaz y transparente por
parte de los gobiernos a todo nivel, mediante el ofrecimiento en línea
de información a los ciudadanos, mecanismos de control de gestión,
servicios, trámites y contratación estatal de bienes y servicios a través
de las redes digitales. (Declaración de Florianópolis, 2000)

Posterior a esta reunión, en la Cumbre de las Américas, celebrada en la ciudad de Quebec en 2001, surgió, por parte de los jefes de Estado y de gobierno presentes, la iniciativa de promover una Agenda de Conectividad como estrategia nacional, regional y subregional, lo cual obtuvo su aprobación mediante Resolución 39 de la Conferencia Mundial de Desarrollo de las Telecomunicaciones. Como resultado de ello, se inició un estudio que buscaba evaluar "el grado de preparación del Gobierno para realizar la transición al Gobierno Electrónico". El estudio tenía tres elementos a evaluar, a saber:

a) si el Estado podía convertirse en un usuario modelo para motivar a otros sectores de la sociedad para que se conecten; b) colocación de información, transacciones y servicios de los Gobiernos en línea; y c) La puesta en marcha de sistemas de adquisición en línea. (CITEL, 2002)

La resolución da origen a la Agenda de Conectividad para las Américas y Plan de Acción de Quito.

Otro de los grandes pasos en la construcción del gobierno electrónico lo constituye la aprobación de la Resolución 56/183 de 2001, por parte de los paí- 
ses miembros de las Naciones Unidas, que convoca a la celebración de la Cumbre Mundial sobre la Sociedad de la Información (CMSI). Esta cumbre se celebró en dos fases: la primera celebrada en Ginebra en el año de 2003. Allí se firmó la Declaración de Principios en la que se expresa la voluntad política para preparar los fundamentos de una sociedad de la información y se estableció un plan de acción que contemplaba entre otros los siguientes objetivos: i) conectar los departamentos de gobierno locales y centrales y crear sitios Web y direcciones de correo electrónico; ii) instruir a los gobiernos en formular políticas nacionales que comprendan estrategias de gobierno electrónico para que la administración pública sea más transparente, eficaz y democrática y finalmente, iii) identificar aplicaciones de las TIC que propicien entornos para la implementación del gobierno electrónico.

La segunda fase fue convocada en Túnez en el año 2005 y concluyó con la Cumbre Mundial sobre la Sociedad de la Información (CMSI). Esta fase tuvo como propósito poner en marcha el Plan de Acción de Ginebra, en especial en la línea de acción número 7 que señaló: "Aplicación de las TIC: beneficios en todos los aspectos de la vida". Adicionalmente, en esta cumbre se alcanzaron acuerdos alrededor de mecanismos de financiación, seguimiento y aplicación de los documentos suscritos en estas dos fases. De esta manera, en Túnez se establece una Agenda para la Sociedad de la Información.

A estas dos conferencias siguieron otras tantas en el entorno latinoamericano como por ejemplo La Conferencia Ministerial Regional preparatoria de América Latina y el Caribe para la Cumbre Mundial sobre la Sociedad de la Información, que se realizó con el auspicio de la CEPAL. Esta conferencia se celebró en las playas Bávaro, Punta Cana, República Dominicana, del 29 al 31 de enero de 2003 y dio origen a la "Declaración de Bávaro".

En la Conferencia Preparatoria Regional Ministerial de América y Latina y el Caribe para la Cumbre Mundial sobre la Sociedad de la Información, celebrada el 10 de junio 2005 en Río de Janeiro, Brasil, se aprobó oficialmente el Plan de Acción Regional eLAC2007. Este Plan se conoce como el Compromiso de Río y concibe El Plan de Acción como una agenda de política pública regionalmente concertada, que reconoce la importancia de las tecnologías de la información y de las comunicaciones (TIC) para la consecución del desarrollo económico y social de los países de la región.

El eLAC2007 (2007) fija, en la meta número 15 con relación con el gobierno electrónico, ocho (8) medidas relacionadas con: (1) cooperación regional, (2) interoperabilidad, (3) ventanillas únicas, (4) firma electrónica/firma digital, (5) modelos de seguridad y preservación de la información, (6) medios de pago electrónico, (7) contratación electrónica y (8) información georeferenciada.

En el año 2006, los Ministros de Relaciones Exteriores y Jefes de Delegación de los Estados miembros de la Organización de los Estados Americanos (ozA), 
reunidos en Santo Domingo, República Dominicana, aprobaron la "Declaración de Santo Domingo: Gobernabilidad y desarrollo en la sociedad del conocimiento".

Finalmente, es importante señalar que el gobierno electrónico alcanza su mayor desarrollo con la "Carta Iberoamericana de Gobierno Electrónico" (2007), que en su preámbulo señala:

Los Ministros de Administración Pública y de la Reforma del Estado y los Jefes de Delegación de los Gobiernos iberoamericanos, reunidos los días 31 de mayo y $1^{\circ}$ de junio de 2007, en Pucón, Chile, en ocasión de la IX Conferencia Iberoamericana de Ministros de Administración Pública y Reforma del Estado renovaron su compromiso con la Reforma del Estado, el fortalecimiento de sus instituciones públicas y la modernización de sus mecanismos de gestión, teniendo en cuenta que la calidad de los organismos públicos es fundamental para el desarrollo, la igualdad de oportunidades y el bienestar social.

La Carta Iberoamericana de Gobierno Electrónico aborda temas relacionados con la simplificación de procedimientos, seguridad, direcciones, sitios, registros, documentos y archivos electrónicos, protección de datos, formación, planificación, interoperabilidad, usabilidad y portales únicos, entre otros muchos temas que son de relevante utilidad para el presente estudio.

En Colombia, el concepto de gobierno electrónico se desarrolla dentro del Programa de Agenda de Conectividad y se denomina Estrategia de Gobierno en Línea, la cual se estableció con carácter obligatoria, de conformidad con lo dispuesto en el Decreto 1151 de 2008.

El programa Agenda de Conectividad: El Salto a Internet representa la reunión de las acciones orientadas a impulsar el desarrollo social y económico de Colombia mediante la masificación de las tecnologías de la información" (CONPES 3072, 2000, pág.12)

El Gobierno en línea es entonces la estrategia del gobierno colombiano en la que adecúa tendencias y estándares internacionales denominadas " $E$ Government"; liderada y orientada por la Organización de Naciones Unidas y cuya finalidad busca la modernización estatal, la implementación de políticas de racionalización de trámites y, sobre todo, el uso de las Tic para permitir de manera efectiva la relación ciudadanos y empresarios con la administración pública. Con el Decreto 1151 de 2008, el Gobierno establece los lineamientos de la implementación de la "Estrategia de Gobierno en Línea de la República de Colombia", cuyo objetivo es contribuir con un Estado más eficiente, transparente, participativo y que preste mejores servicios a los ciudadanos y a las empresas, mediante el beneficio de las Tecnologías de la Información y la Comunicación. 


\section{Mecanismos jurídico - administrativos que propician la flexibilización de la gestión del Estado}

Se hace inminente identificar y analizar los principales cambios requeridos que integren eficientemente las TIC, acerquen al país al mundo globalizado y, a su vez, contribuyan a constituir escenarios propios para el surgimiento de nuevos estilos de gestión organizacional que influencien los procesos de acercamiento entre el Estado y la sociedad civil. Para lograr este propósito, el Estado debe estar dispuesto a implementar mecanismos jurídicos que hagan posible romper la rigidez de su estructura y permitan que en la toma de decisiones políticas, cada ciudadano o grupo representativo de la sociedad civil sean los verdaderos y auténticos protagonistas.

Las nuevas tecnologías incorporadas a la gestión pública se constituyen en motor para el desarrollo de los Estados. Poner a disposición de los particulares un gobierno electrónico significa que los servicios gubernamentales están pensados para dar respuestas efectivas, prontas, seguras e incluyentes a los ciudadanos.

Dentro de este concepto de inclusión, el gobierno electrónico se constituye en la forma pertinente para responder a las crecientes demandas de información y servicios gubernamentales que denoten neutralidad y transparencia en el hacer del Estado. Finalmente, y como una etapa de mayor adelanto, habilita la posibilidad de exigir y recibir rendición de cuentas de los funcionarios. El gobierno electrónico se proyecta, hacia el futuro, como el impulsador de las expectativas de lo que los gobiernos pueden y deben hacer con el apoyo de las TIC y que han de concretarse en el fortalecimiento de los servicios públicos y del desarrollo equitativo centrado en las personas.

Por otra parte, la implementación del e-gobierno plantea grandes retos entre los cuales se destacan los siguientes: i) la reducción de la denominada brecha digital; ii) el aumento del acceso a los servicios públicos en poblaciones vulnerables y comunidades alejadas, como consecuencia del uso de la información en la nube; iii) la implementación de servicios más prácticos, como los quioscos de servicios electrónicos y; iv) la inclusión de otros servicios que hagan posible sustentar, fortalecer y poner a disposición de todos los segmentos de la sociedad la gestión del Estado.

A continuación se presentan algunos mecanismos que fueron identificados en el desarrollo de la presente investigación como respuesta a los objetivos propuestos en la misma, veamos:

a. Creación de nuevas formas de administración del Estado en las que se propicia la descentralización territorial y funcional

Al Estado siempre se le ha criticado la forma centralista como pretende dar solución a los problemas del país. Es común encontrar en las reuniones de alcaldes o gobernadores, agrupados en asociaciones, la solicitud de mayor presencia del 
Estado en zonas en donde no se cumple con las funciones fundamentales del mismo y ver cómo este fenómeno se convierte en un factor que propicia la presencia de grupos alzados en armas que, con su actuar, suplen la institucionalidad. Al respecto, una solución sería buscar nuevas formas de ejercer la administración dentro de un enfoque descentralizador que le den vida a las regiones como forma de organización consagrada en la Constitución. Sería un modelo intermedio entre las gobernaciones y el ejecutivo, de tal suerte que otorgue a esos nuevos entes territoriales una organización no piramidal, sino colaborativa, facultándolas para hacer uso de metodologías gerenciales que permitan reacciones inmediatas y cercanas a la comunidad.

Se desprende de la propuesta anterior que, reconociendo la necesidad de que sea inmediata la pérdida de rigidez de la estructura del Estado con el fin de otorgar adecuados procesos administrativos en la etapa del posconflicto al que está llegando el país, dentro del proceso de paz que adelanta Colombia, se debe propiciar una mayor autonomía de los municipios y departamentos para dar soluciones a los intereses de las comunidades dentro de cada uno de estos entes territoriales. En efecto, una mayor descentralización o autonomía de estos entes exige dotarlos efectivamente de los recursos para que ello sea posible.

De todo lo dicho podemos concluir que, si bien se hacen necesarias nuevas formas de participación social, éstas deben integrarse como suma junto a la responsabilidad que el Estado ya tiene y no encauzarse a restarle parte de su responsabilidad en la búsqueda de una mayor equidad en la región, clave para el desarrollo. (Barone, 2001, p.1)

\section{b. Ampliación de la oferta de servicios gubernamentales en línea}

Se necesita crear nuevos esquemas de administración pública, resultado de los adelantos en las tecnologías de la información y las comunicaciones, que permitan desarrollar mecanismos de respuesta a las necesidades de la sociedad, de tal forma que hagan posible la vinculación de nuevos actores políticos y sociales y surjan acuerdos explícitos e implícitos, de larga duración, entre estos actores y el Estado, en torno a la participación ciudadana tendiente a dar soluciones a las inquietudes que emanen sobre los servicios del Estado; se deben crear espacios para debatir las inquietudes sobre la definición de políticas públicas de trascendencia social y se debe dar cabida a manifestarse sobre las acciones de la administración en general para que emerjan procesos efectivos de seguimiento y evaluación.

En este orden de ideas y de acuerdo con el Manual para la implementación de la Estrategia Gobierno en Línea en las entidades del orden nacional de la República de Colombia (MinTic, 2012) la participación de la sociedad en general se consolida como el derecho de los ciudadanos a participar en la construcción de lo público y 
de las organizaciones para abrirse a la sociedad y mostrar sus problemas propiciando la acción de actores que tengan soluciones disponibles en menor tiempo y a mejores costos.

En este mismo sentido se expresa el profesor Navarro (1998):

[P]or lo tanto en concordancia con este papel relevante que se le asigna a la sociedad civil, las organizaciones que conforman el Estado se deben trasformar en la misma dirección, es decir, se deben organizar privilegiando como núcleo de la estructura y de su proceso de trabajo a los usuarios y clientes de sus productos y servicios tanto internos como externos. (p. 6)

La revolución digital no puede excluir parte de la sociedad y es allí en donde la labor del Estado, como promotor de la industria privada, debe generar instrumentos que le permitan a las empresas privadas ofrecer los avances de las comunicaciones, en condiciones favorables, sin desfavorecer, por costo, a una parte de los posibles usuarios.

La llamada "brecha digital", como concepto, fue introducido a mediados de la década de los noventa y se presentó como una brecha en el desarrollo y no como una brecha en la infraestructura. La Declaración de la Cumbre sobre la Sociedad de la Información ${ }^{2}$, en la que se asume la temática de la sociedad de la información y de su estudio, propone múltiples estrategias para lograr que todos los países en desarrollo se beneficien de las nuevas TIC (ONU, 2005).

La legislación colombiana consagra el Derecho a la Información como una norma de rango constitucional, en donde se constituye, dentro de la estrategia de gobierno en línea, como condición básica para propiciar la transparencia en la gestión pública y con ello reducir los riesgos de corrupción, pues eleva los niveles de transparencia. Esta última, a su vez, aporta elementos al cuidado de los recursos públicos y genera condiciones de afianzamiento de la relación de los diferentes actores con la administración pública, elemento clave para la gobernabilidad y la creación de capital social. A propósito el MinTIC precisa:

Adicionalmente la información que poseen las entidades conforma un bien público, el cual puede ser utilizado por terceros en la creación de valor para la ciudadanía en general a través de esquemas de datos abiertos, los cuales ofrecen una ventana de oportunidad para la generación de más y mejores servicios apalancados en las condiciones del mercado. (MinTIC, 2012, p.22)

2 Declaración que se suscribió producto de la Cumbre Mundial sobre la Sociedad de la Información (CMSI) realizada en diciembre de 2003, en Ginebra, y la adelantada en noviembre del 2005, en Túnez. 
De igual manera la implementación del nuevo modelo de Gobierno en línea implica para las entidades la alineación de actividades con otros temas esenciales de la gestión pública en Colombia, como lo son: la Política Antitrámites, la Política Nacional del Servicio al Ciudadano, la Política de Rendición de Cuentas a la Ciudadanía, la Política Nacional Anticorrupción, la Política Nacional de Archivo y Gestión Documental, entre otras. (MinTIC, 2012, p.5)

\section{c. Participación efectiva de la ciudadanía en la toma de decisiones gubernamentales con el uso de plataformas digitales y redes sociales}

La participación de la sociedad en la construcción de su propia institucionalidad debe ser cada vez más activa, dejando de lado los conceptos propios de regímenes centralistas, dando prelación, en forma real y efectiva, a las mayorías sociales. Es función prioritaria del Estado legitimar la participación, estimular la cultura política y eliminar la apatía ciudadana, producto de la desconfianza y descredito hacia la clase política.

El profesor Castells (1998), refiriéndose a la participación democrática, afirma:

La participación no es la democracia. La democracia no es un principio de reforma de la administración porque es un principio político establecido y que se da por sobre-entendido en nuestro contexto. Podría incluso pensarse que sin democracia los estados nación se hacen inviables a medio plazo en un contexto mundial que plantea constantes desafíos. (pág.13)

Más adelante, el profesor Castells se manifiesta en el sentido de considerar que los ciudadanos articulados con los Estados, mediante el uso de una comunicación fluida, pueden generar debates que integren asuntos que afectan directamente a la comunidad. Finalmente expresa:

Sin participación, la democracia se irá vaciando de contenido para amplios sectores de la población, sobre todo para los 'sin voz', cuya carencia de recursos materiales y culturales, en ausencia de procesos participativos, les condena a ser comparsas o enrabiados (pág.13).

Incentivar la participación ciudadana en la toma de decisiones gubernamentales exige el uso eficiente de plataformas digitales, redes sociales y herramientas que permitan el intercambio electrónico de información y conocimiento tales como el correo electrónico, las conferencias electrónicas, la firma electrónica, la contratación por medios electrónicos o la notificación de actos administrativos en forma virtual. En consonancia, propiciar todo aquello que las tecnologías de la 
información y las telecomunicaciones faciliten para suscitar el debate y la puesta en común de soluciones viables a los problemas específicos de la sociedad o hagan viable otros servicios propios del Estado como, por ejemplo, trámites administrativos o sistemas de quejas y reclamos.

El gobierno colombiano, en desarrollo de la Estrategia del Gobierno en Línea, considera que:

\begin{abstract}
El rol de los ciudadanos también ha venido transformándose, las formas de expresión son diversas y se basan en mecanismos más directos y más poderosos, la mayoría apoyados en el uso de las Tecnologías de la Información y las Comunicaciones-TIC. La ciudadanía tiene un conocimiento importante que puede ser aprovechado en beneficio de las sociedades y el Estado puede servir de plataforma para canalizar y potenciar dicho conocimiento. Asimismo, son los ciudadanos los beneficiarios directos de las políticas públicas y de la toma de decisiones, lo que hace cada vez más imperante involucrarlos activamente en su construcción y validación. (MinTIC, 2012, p.4)
\end{abstract}

En el contexto de la implementación del gobierno electrónico, los estados tienen la labor de propiciar cambios en su forma de operar y gestionar, de tal suerte que se garantice una mejor y fácil comunicación entre éstos y el ciudadano. Optimizar la comunicación ha de tener como metas las siguientes: i) hacer más eficiente la gestión pública; ii) mejorar la satisfacción de los usuarios; iii) proteger y optimizar los recursos públicos y iv) promover y hacer más y mejor control social.

A propósito de lo expuesto, se trascribe la definición de democracia en línea propuesta por el MinTIC:

Democracia en línea: comprende todas las actividades para que las entidades creen un ambiente para empoderar a los ciudadanos e involucrarlos en el proceso de toma de decisiones. Con estas actividades se propicia que el ciudadano participe activa y colectivamente en la toma de decisiones de un Estado totalmente integrado en línea. Igualmente, se promueve que las entidades públicas incentiven a la ciudadanía a contribuir en la construcción y seguimiento de políticas, planes, programas, proyectos, la toma de decisiones, el control social y la solución de problemas que involucren a la sociedad en un diálogo abierto de doble vía. (MinTIC, 2012, p.8)

\title{
4. Mecanismos jurídico-administrativos implementados por el gobierno colombiano dentro de la estrategia de gobierno en línea
}

En cumplimiento de los compromisos internacionales sobre la implementación del gobierno electrónico, el Estado colombiano ha venido adoptando, desde el 
año 2000, políticas públicas que buscan conseguir un "buen gobierno" y en ese marco institucional adoptó la Estrategia de Gobierno en Línea que se constituyó en un instrumento de utilidad para modernizar la administración pública y hacer uso de los avances vertiginosos de las TIC, en este sentido puso al servicio de la ciudadanía una serie de programas que se aplican a todas las ramas del poder público y a todos los niveles territoriales.

De acuerdo con el Ministerio de las TIC, en Colombia existen cerca de 1.900 posibilidades de realizar operaciones, tramitar, solicitar información o servicios con el Estado, facilitados por los medios electrónicos. Entre estas soluciones se puede resaltar:

a. El sistema de pagos de aportes a la seguridad social, por medio del aplicativo tecnológico de transferencia electrónica de fondos denominado Planilla Integrada de Liquidación de Aportes (PILA), dirigido a trabajadores independientes y empresas.

b. El Registro Único Nacional de Tránsito (RUNT) para asuntos relacionados con los automotores.

c. El Portal Único de Contratación (PUC).

d. El Sistema Electrónico para la Contratación Pública (SECOP).

e. El programa Colombia Compra Eficiente, que permite la optimización de la compra de productos y servicios por parte del Estado.

f. El Plan Vive Digital que impulsa la masificación del uso de internet con el fin de reducir la pobreza, generar empleo y que todos los colombianos cuenten con un espacio virtual en el que puedan acceder a su correo electrónico, documentos oficiales, formularios de pago de impuestos. También permite solicitar certificados de propiedad, conocer la historia médica, obtener el Certificado Judicial y los antecedentes disciplinarios y fiscales.

g. El programa Caja de Cristal en el que los ciudadanos pueden realizar preguntas y propuestas a los entes gubernamentales.

La Agenda de Conectividad definió, por primera vez, los componentes tecnológicos que constituirían el gobierno en línea. Uno de ellos se refiere a la plataforma de servicios que el Gobierno nacional puede brindarle a los ciudadanos, por medio de la plataforma web, desde donde se desprenderán los sitios web de las entidades públicas. Este componente se denomina Portal del Estado Colombiano (PEC) y está vinculado con el sitio web: www.gobiernoenlinea.gov.co, ello permite el acceso de los ciudadanos a la información oficial y servicios que el Estado presta a través de medios virtuales. Otro componente fundamental se refiere a intranet o comunicación entre las instituciones estatales. 
En el Manual de Políticas y Estándares para la Gestión de información, Trámites y Servicios del Estado Colombiano a través de Medios Electrónicos (MinTIC, 2012) se fijaron los protocolos para que las entidades públicas dispongan en línea de diversos trámites y servicios. Así mismo, se impuso una plantilla para el diseño de los sitios web y se estableció un único lenguaje o código de programación, el GEL-XML (Gobierno en Línea Extensible Markup Language).

En las páginas web de cada una de las entidades se debe cumplir con la Resolución No. 3564 de 2015, Por la cual se reglamentan aspectos relacionados con la Ley de Transparencia y Acceso a la Información Pública, Anexo 1 Estándares para publicación y divulgación de información (Colombia, MinTIC, 2015), la cual de manera general identifica los aspectos centrales y las posibles herramientas que permiten al ciudadano tener conocimiento de la información pertinente de las entidades estatales. Al respecto, se espera que la entidad informe los canales de comunicación para los ciudadanos, usuarios y grupos de interés dispuestos en medios virtuales.

El uso de medios virtuales pretende, en primer lugar, que el Estado colombiano sea eficiente, transparente y participativo $y$, adicionalmente, que ofrezca mejores y oportunos servicios a la sociedad. De modo que el desarrollo de la estrategia gobierno en línea se desarrolla bajo tres ejes de acción aplicables tanto a las ramas del poder público, como a todos los tres niveles territoriales. Esos ejes de acción son: mejorar los servicios a los ciudadanos y a las empresas; fortalecer la transparencia del Estado y participación ciudadana y, tercero, mejorar la eficiencia del Estado.

De conformidad con el Plan Estratégico de Optimización y Automatización de Cadenas de Trámites en la Administración Pública Colombiana, aprobado por el Ministerio de las TIC (MinTIC, 2009), los componentes que identifican cada uno de los ejes son:

a. Mejorar los servicios a los ciudadanos y a las empresas: ofrecer servicios que realmente respondan a las necesidades de los ciudadanos y las empresas; optimar la calidad de dichos servicios provistos por el Estado; ahorrar costos y tiempo para el ciudadano; atender de manera unificada al ciudadano; incrementar la satisfacción de los ciudadanos en su interacción con el Estado; simplificar los trámites que los ciudadanos deben realizar ante el Estado y promover el acceso a los servicios del Estado a través de múltiples canales.

b. Fortalecer la transparencia del Estado y la participación ciudadana: incrementar la visibilidad de los asuntos públicos (oferta de información y rendición de cuentas); facilitar el conocimiento de la gestión del Estado, mejorar la confianza en la gestión del Estado; crear nuevos canales que faciliten la participación ciudadana y el control de la gestión pública y 
facilitar la interacción ciudadano/Estado y obtener retroalimentación de los ciudadanos.

c. Mejorar la eficiencia del Estado: perfeccionar la calidad, oportunidad y estandarización de la información para el diseño, planeación, ejecución y evaluación de las políticas públicas y racionalizar y optimizar los recursos del Estado.

\section{Conclusiones}

A manera de conclusión, la importancia del presente estudio consiste en describir las obligaciones que se le imponen al Estado a raíz de la incorporación de las TIC en la administración del mismo, sin perder de vista que el acceso a estas es un derecho fundamental reconocido en el artículo número 27 de la Declaración de los Derechos Humanos en 1946, y que dice: "Toda persona tiene derecho a tomar parte libremente en la vida cultural de la comunidad, a gozar de las artes y a participar en el progreso científico y en los beneficios que de él resulten" (ONU).

No obstante lo anterior, la investigación ha permitido entender que el Estado debe recuperar su rol central en la vida pública, superar aquella idea de reducir el tamaño del mismo y limitar su actividad solo a temas sociales y, en particular, los servicios para los más pobres. Cuando se afirma que se trata de recuperar el rol central, se hace alusión a que el Estado tiene una responsabilidad fundamental: ser el responsable de garantizar el efectivo acceso a los derechos fundamentales por parte de los ciudadanos.

No se puede desconocer que los procesos de globalización, acompañados de los cambios en las tecnologías de la información y de las comunicaciones, exigen una verdadera redefinición de las funciones del Estado, transformándolo de mero administrador de los intereses públicos a uno que propicie la participación de los ciudadanos en los procesos de definición de las políticas públicas, con miras a lograr una auténtica democracia participativa que tenga como norte los principios constitucionales de transparencia, calidad, universalidad, bien común y demás aspectos esenciales del Estado de Derecho, que la verdad sea dicha, en el caso colombiano, aun no son claros y contundentes y las acciones gubernamentales solo se practican como reacción a exigencias del mercado y no como fruto de una autentica visión de gobierno en búsqueda de los fines esenciales del Estado.

La transformación del Estado colombiano es un imperativo ineludible, no solo como organización prestadora de servicios a los ciudadanos, sino también como ente dinamizador de la sociedad en general que permita procesos de reingeniería que le hagan competitivo en el marco de las exigencias de una economía globalizada, con la participación activa de los particulares. 
El gobierno electrónico debe propender por concretar ideales administrativos como la calidad y eficacia de los servicios que presta a los ciudadanos, la participación de la comunidad en general e incentivar una mayor confianza en la labor del Estado, como garante del logro de los objetivos superiores de la administración pública, que se concretan en los fines esenciales del Estado, plasmados en la Constitución Política.

La estrategia gobierno en línea en Colombia ha permitido grandes beneficios en las tres ramas del poder público del Estado y a todos los niveles territoriales. Dentro de esos logros se pueden señalar: el mejoramiento en los tiempos de respuesta de los trámites y servicios, la calidad de la información que se brinda en las páginas web, la apertura de escenarios propios para que la ciudadanía participe activamente en el ejercicio de control de la actividad administrativa o en la definición de políticas públicas. Así mismo, se han implementado otras formas de participación como: poder presentar observaciones sobre el nombramiento de funcionarios que dependen de la Presidencia de la República, cargos sometidos al análisis de los interesados. No obstante, quedan pendientes grandes transformaciones, como, por ejemplo: el voto electrónico, la participación efectiva de las comunidades en la discusión de los presupuestos en todos los ámbitos de la administración, la eliminación de trámites y el fortalecimiento de los formularios en línea para la apertura de nuevas sociedades y terminación de las que no son exitosas, entre otros.

Los aspectos señalados anteriormente se han convertido en verdaderas políticas públicas que articulan la gestión de todos los entes del Estado y que se hacen visibles dentro de la estrategia de gobierno en línea. Algunas de ellas son: la Política Antitrámites, la Política Nacional del Servicio al Ciudadano, la Política de rendición de cuentas a la ciudadanía, la Política Nacional Anticorrupción, la Política Nacional de Archivo y Gestión Documental.

La investigación desarrollada para evaluar los mecanismos jurídico-administrativos que pueden ser implementados por el Estado colombiano para darle acceso a obtener una estructura administrativa más flexible y con ello responder, en forma oportuna, a las exigencias originadas en las políticas públicas que atienden los fenómenos de la internacionalización y la globalización, dentro del marco de gobierno electrónico o gobierno en línea, permiten afirmar, de forma argumentada, que el abanico de alternativas propuestas dan cuenta de cómo las TIC propician la implementación de mecanismos jurídico - administrativos y hacen efectivos los servicios que el Estado presta a los ciudadanos en cumplimiento con las funciones públicas enmarcadas en principios constitucionales y legales que imponen la construcción de una administración estatal democrática, solidaria y participativa. Pero quedan muchas otras más para poder garantizar una verdadera modernización de la actividad del Estado y el aseguramiento del ejercicio fundamental a gozar de los adelantos científicos y tecnológicos. 


\section{Referencias}

Albornoz, M. B. (2006). Versiones de Gobierno Electrónico implementadas por el Municipio de Quito. Informe ganador de Ecuador en el concurso de investigación Experiencias andinas de gobierno electrónico: la problemática de la participación ciudadana, organizado por FLACSO Ecuador y financiado por el Centro Internacional de Investigación para el Desarrollo (IDRC-CDRI) Ottawa, Canadá.

Barone, M. (2001). Globalización y posmodernidad: encrucijada para las políticas sociales del nuevo milenio. Universidad de Sao Paulo. Recuperado de: http://www.cepal. org/mujer/noticias/noticias/0/8260/posmodernidad.pdf

Castells, M (1996). The Information Age. Blackwell Publishers.

Castells, M (1998). Hacia el Estado Red. Globalización económica e instituciones políticas en la era de la información. Ponencia presentada en el Seminario sobre "Sociedad y reforma del Estado", organizado por el Ministerio de Administracao Federal e Reforma Do Estado, República Federativa do Brasil. Sao Paulo, 26-28 marzo 1998.

CEPAL/CLADES, (1995). Los sistemas de información y el desafío de los cambios globales. Serie Información y Desarrollo Casilla 179-D, Santiago de Chile. Recuperado de: http://repositorio.cepal.org/bitstream/handle/11362/9695/ S9500142_es.pdf?sequence=1\&isAllowed=y

Comisión Interamericana de Telecomunicaciones (CITEL). (2003). Agenda de Conectividad para las Américas y Plan de Acción de Quito, pp 54

Centro Latinoamericano de Administración para el Desarrollo (CLAD). (2007). Carta Iberoamericana de Gobierno Electrónico. Adoptada por la XVII Cumbre Iberoamericana de Jefes de Estado y de Gobierno Santiago de Chile, 10 de noviembre de 2007. Recuperado de: http://old.clad.org/documentos/declaraciones/cartagobelec.pdf/view

Colombia. Consejo de Estado. Sentencia 763 de (2011).

Colombia. Ministerio de las T.I.C. (2015). Resolución No. 3564. Por la cual se reglamentan aspectos relacionados con la Ley de Transparencia y Acceso a la Información Pública", Anexo 1, "Estándares para publicación y divulgación de información", Diario Oficial No. 49.829 de 30 de marzo de 2016

Colombia. Ministerio de Comunicaciones. (2019). Plan Nacional de Tecnologías de la Información y las Comunicaciones. República de Colombia.

Colombia. Ministerio de las T.I.C. (2012). Manual para la implementación de la Estrategia de Gobierno en Línea en las entidades del orden nacional de la República de Colombia, Bogotá, pp.94

Colombia. Ministerio de las T.I.C. (2011). Manual 3.0 para la implementación de la estrategia de gobierno en línea en las entidades del orden nacional de la república de Colombia, Bogotá, 2011, pp.48 
Colombia. Ministerio de la T.I.C. (2009). Plan estratégica de optimización y automatización de cadenas de trámites en la administración pública colombiana República de Colombia, Bogotá, 2009, pp.108

Colombia. Ministerio de la T.I.C. (2000). CONPES 3072. Agenda de Conectividad, Bogotá.

Cooperación RED (2009). Redes para el Desarrollo Humano: Redes globales emergentes. Grupo de trabajo C-56. Recuperado de: http://www.cibersociedad.net/ congres2009/es/coms/redes-globales-emergentes/137/

Cortés Sánchez, J. D. \& Cardona Madariaga, D. F. (2015). Gobierno Electrónico en América Latina - Revisión y Tendencias. Ed. Universidad del Rosario, Bogotá, pp.114

Drucker P. (1994). La Sociedad Postcapitalista. Bogotá. Norma.

Durán, V. M. (2001). Estado Social de Derecho, Democracia y Participación. Ponencia: VII Conferencia Latinoamericana de Trabajadores de los Servicios Públicos. México, 22-25 de abril de 2001. Recuperado de: https://upload.wikimedia.org/ wikipedia/commons/4/44/Estado_Social_de_Derecho\%2C_Democracia_y_ Participaci\%C3\%B3n.pdf

Enríquez Pérez, I. (2002). Los procesos de globalización y la política pública como instrumento reestructurador del espacio urbano en México. Revista Economía, sociedad y cultura, enero de 2002. Recuperado de: http://rcci.net/globalizacion.

IV Congreso de la cibersociedad (2009). Crisis analógica, futuro digital. Grupo de trabajo C-56: Redes para el Desarrollo Humano: la Cooperación Red. Recuperado de: http://www.cibersociedad.net/congres2009/es/gts/redes-para-el-desarrollohumano-la-cooperacion-red/56/

Naser, A. \& Concha, G. (2011). El gobierno electrónico en la gestión pública. Editorial CEPAL, ILPES. Santiago de Chile, pp. 41. Recuperado de: http://www.cepal.org/ilpes/noticias/paginas/3/54303/El_Gobierno_electronico_en_la_gestion_Publica.pdf

Navarro Vargas, Á. F. (1998). Gerencia Pública y Modernización del Estado. Revista Costarricense de Trabajo Social, núm., pp. 59 - 67. Recuperado de: http://www. ts.ucr.ac.cr/binarios/docente/pd-000174.pdf

Organización de Naciones Unidas (ONU). (2008). UN E-Government Survey 2008. Nueva York. Recuperado de: http://www.unpan.org/egovkb/global_reports/08report.htm

Organización de Naciones Unidas (ONU). (2012). Resumen Ejecutivo. En: Estudio de las Naciones Unidas sobre el Gobierno Electrónico, 2012 Gobierno electrónico para el pueblo, 2012. Nueva York, pp. 1-9.

Ospina Bozzi, S. M. (2000). Globalización y Desarrollo local: hacia una perspectiva municipalista. Ponencia plenaria V Congreso Iberoamericano de Municipalistas. Baeza, España.

Programa de Naciones Unidas para el Desarrollo (PNUD). (2009). AGENDA DE CONECTIVIDAD, UNIVERSIDAD DE LOS ANDES, Informe de Gobierno en Línea 2008-2009, Recuperado de: http://programa.gobiernoenlinea.gov.co 
Suelt Cock, V. (2008). La organización territorial colombiana frente a la tendencia de configuración federal. Revista de Estudios Socio Jurídicos 1, 155-200. Recuperado de: http://www.javeriana.edu.co/pesquisa/wp-content/uploads/pesquisa32_04.pdf

TELECOM (1990). Las telecomunicaciones en el gobierno de Virgilio Barco 86-90. Empresa Nacional de Telecomunicaciones. Bogotá.

Velázquez Becerril, C. A. \& Pérez Pérez, G. (2010). Las transformaciones del Estado-nación en el contexto de la globalización. Política y Cultura, otoño 2010, núm. 33, pp. 107-127

Vieira Silva, J. G. (2008). Administración Pública para el Desarrollo Integral: Una Cuestión de Interés Público. Recuperado de: https://www.academia.edu/7335500/ADMINISTRACION_PUBLICA_PARA_EL_DESARROLLO_INTEGRAL_UNA_CUESTION_DE_INTERES_PUBLICO. 